\title{
Supplementary Information: Antimicrobial and antibiofilm activities of helical antimicrobial peptide sequences incorporating metal-binding motifs
}

Caleb M. Agbale $1,7,8$, Justice K. Sarfo ${ }^{1}$, Isaac K. Galyuon ${ }^{8}$, Samuel A. Juliano ${ }^{3}$, Gislaine G. O. Silva $^{1}$, Danieli F. Buccini ${ }^{1}$, Marlon H. Cardoso ${ }^{1,5,6}$, Marcelo D. T. Torres ${ }^{2}$, Alfredo M. AngelesBoza $^{3,4}$, Cesar de la Fuente-Nunez ${ }^{2, *}$ and Octavio L. Franco ${ }^{1,5,6, *}$

${ }^{1}$ S-INOVA Biotech, Programa de Pós-Graduação em Biotecnologia, Universidade Católica Dom Bosco, Campo Grande, Mato Grosso Do Sul, MS, Brasil.

${ }^{2}$ Machine Biology Group, Departments of Psychiatry and Microbiology, Perelman School of Medicine, and Department of Bioengineering, University of Pennsylvania, Philadelphia, Pennsylvania, United States of America.

${ }^{3}$ Department of Chemistry, University of Connecticut, Storrs, CT, United States of America.

${ }^{4}$ Institute of Materials Science, University of Connecticut, Storrs, CT, United States of America.

${ }^{5}$ Centro de Análises de Proteômicas e Bioquímicas, Programa de Pós-Graduação em Ciências Genômicas e Biotecnologia, Universidade Católica de Brasília, Brasília, DF, Brasil.

${ }^{6}$ Programa de Pós-Graduação em Patologia Molecular, Faculdade de Medicina, Universidade de Brasília, Brasília, DF, Brazil.

${ }^{7}$ Department of Biochemistry, School of Biological Sciences, College of Agriculture and Natural Sciences, University of Cape Coast, Ghana.

${ }^{8}$ Department of Molecular Biology and Biotechnology, School of Biological Sciences, College of Agriculture and Natural Sciences, University of Cape Coast, Ghana.

Correspondence:

Cesar de la Fuente-Nunez (cfuente@pennmedicine.upenn.edu);

Octavio L. Franco (ocfranco@gmail.com). 
Table S1. The list of $\alpha$-helical AMPs from the antimicrobial peptide database (http://aps.unmc.edu/AP/database/antiA.php) selected in silico for this study. For subsequent evaluation, the tripeptide motifs GGH and VIH were inserted into the N-terminal extremity of the peptides.

\begin{tabular}{|c|c|c|c|c|}
\hline Label & APD ID & $\begin{array}{l}\text { Sequence } \\
\text { length }\end{array}$ & Name & Sequence* \\
\hline 1 & 2531 & 17 & Stigmurin & FFSLIPSLVGGLISAFK \\
\hline 2 & 2447 & 16 & Scolopendin 2 & AGLQFPVGRIGRLLRK \\
\hline 3 & 2409 & 20 & Salusin- $\beta$ & AIFIFIRWLLKLGHHGRAPP \\
\hline 4 & 2386 & 19 & P2-Hp-1935 & KLSPSLGPVSKGKLLAGQR \\
\hline 5 & 1637 & 17 & Pm_mastoparan PMM & INWKKIASIGKEVLKAL \\
\hline 6 & 1545 & 18 & Meucin-18 & FFGHLFKLATKIIPSLFQ \\
\hline 7 & 1516 & 15 & Eumenitin & LNLKGIFKKVASLLT \\
\hline 8 & 1466 & 15 & Lasioglossin LL-II & VNWKKILGKIIKVAK-NH ${ }_{2}$ \\
\hline 9 & 1461 & 16 & Alyteserin-2a & ILGKLLSTAAGLLSNL-NH $_{2}$ \\
\hline 10 & 1223 & 19 & Ascaphin-8 & GFKDLLKGAAKALVKTVLF-NH \\
\hline 11 & 1007 & 15 & CM15 & KWKLFKKIGAVLKVL \\
\hline 12 & 427 & 18 & CPF-ST3 & GLLGPLLKIAAKVGSNLL-NH $_{2}$ \\
\hline 13 & 381 & 18 & Ponericin G6 & GLVDVLGKVGGLIKKLLP-NH $_{2}$ \\
\hline 14 & 351 & 16 & Citropin1.1 & GLFDVIKKVASVIGGL-NH$_{2}$ \\
\hline
\end{tabular}

* $-\mathrm{NH}_{2}$ indicates amidated C-terminal extremity. 
Table S2. Output data of prediction algorithms. The modules available at SVM: support vector machine; DA, discriminant analysis; RF, random forest; ANN, artificial neural network.

\begin{tabular}{|c|c|c|c|c|c|c|}
\hline \multirow{2}{*}{ Peptide } & \multirow{2}{*}{ Sequence } & \multicolumn{4}{|c|}{ AMP Prediction Algorithm } & \multirow{2}{*}{ DBAASP } \\
\hline & & SVM & RF & ANN & DA & \\
\hline Stigmurin & FFSLIPSLVGGLISAFK & 0.942 & 0.954 & AMP & 0.976 & AMP \\
\hline GGH-Stigmurin & GGHFFSLIPSLVGGLISAFK & 0.943 & 0.850 & AMP & 0.989 & AMP \\
\hline VIH-Stigmurin & VIHFFSLIPSLVGGLISAFK & 0.927 & 0.840 & AMP & 0.966 & AMP \\
\hline Scolopendin 2 & AGLQFPVGRIGRLLRK & 0.947 & 0.969 & AMP & 0.990 & AMP \\
\hline GGH-Scolopendin 2 & GGHAGLQFPVGRIGRLLRK & 0.953 & 0.738 & AMP & 0.996 & AMP \\
\hline VIH-Scolopendin 2 & VIHAGLQFPVGRIGRLLRK & 0.943 & 0.989 & AMP & 0.998 & AMP \\
\hline Salusin- $\beta$ & AIFIFIRWLLKLGHHGRAPP & 0.926 & 0.839 & AMP & 0.973 & AMP \\
\hline GGH-Salusin- $\beta$ & GGHAIFIFIRWLLKLGHHGRAPP & 0.941 & 0.881 & AMP & 0.994 & AMP \\
\hline VIH-Salusin- $\beta$ & VIHAIFIFIRWLLKLGHHGRAPP & 0.886 & 0.851 & AMP & 0.983 & AMP \\
\hline P2-Hp-1935 & KLSPSLGPVSKGKLLAGQR & 0.554 & 0.801 & AMP & 0.705 & AMP \\
\hline GGH-P2-Hp-1935 & GGHKLSPSLGPVSKGKLLAGQR & 0.766 & 0.827 & AMP & 0.961 & AMP \\
\hline VIH-P2-Hp-1935 & VIHKLSPSLGPVSKGKLLAGQR & 0.813 & 0.970 & AMP & 0.982 & AMP \\
\hline Pm_mastoparan PMM & INWKKIASIGKEVLKAL & 0.997 & 0.982 & AMP & 0.992 & AMP \\
\hline $\begin{array}{c}\text { GGH-Pm_mastoparan } \\
\text { PMM }\end{array}$ & GGHINWKKIASIGKEVLKAL & 0.981 & 0.955 & AMP & 0.993 & AMP \\
\hline $\begin{array}{c}\text { VIH-Pm_mastoparan } \\
\overline{\text { PMM }}\end{array}$ & VIHINWKKIASIGKEVLKAL & 0.986 & 0.972 & AMP & 0.996 & AMP \\
\hline Meucin-18 & FFGHLFKLATKIIPSLFQ & 0.961 & 0.977 & AMP & 0.946 & AMP \\
\hline GGH-Meucin-18 & GIHFFGHLFKLATKIIPSLFQ & 0.962 & 0.890 & AMP & 0.974 & AMP \\
\hline VIH-Meucin-18 & VIHFFGHLFKLATKIIPSLFQ & 0.931 & 0.917 & AMP & 0.953 & AMP \\
\hline Eumenitin & LNLKGIFKKVASLLT & 0.989 & 0.984 & AMP & 0.994 & AMP \\
\hline GGH-Eumenitin & GGHLNLKGIFKKVASLLT & 0.981 & 0.961 & AMP & 0.996 & AMP \\
\hline VIH-Eumenitin & VIHLNLKGIFKKVASLLT & 0.986 & 0.998 & AMP & 0.998 & AMP \\
\hline Lasioglossin LL-II & VNWKKILGKIIKVAK-NH & 0.996 & 0.999 & AMP & 0.996 & AMP \\
\hline GGH-Lasioglossin LL-II & GGHVNWKKILGKIIKVAK-NH ${ }_{2}$ & 0.994 & 0.986 & AMP & 0.999 & AMP \\
\hline VIH-Lasioglossin LL-II & VIHVNWKKILGKIIKVAK-NH ${ }_{2}$ & 0.993 & 0.995 & AMP & 1.000 & AMP \\
\hline Alyteserin-2a & ILGKLLSTAAGLLSNL-NH $_{2}$ & 0.961 & 0.735 & AMP & 0.987 & AMP \\
\hline GGH-Alyteserin-2a & GGHILGKLLSTAAGLLSNL-NH & 0.943 & 0.736 & AMP & 0.989 & AMP \\
\hline VIH-Alyteserin-2a & VIHILGKLLSTAAGLLSNL-NH ${ }_{2}$ & 0.969 & 0.972 & AMP & 0.992 & AMP \\
\hline Ascaphin-8 & GFKDLLKGAAKALVKTVLF-NH $_{2}$ & 0.990 & 0.971 & AMP & 0.999 & AMP \\
\hline GGH-Ascaphin-8 & GGHGFKDLLKGAAKALVKTVLF-NH ${ }_{2}$ & 0.975 & 0.962 & AMP & 1.000 & AMP \\
\hline
\end{tabular}




\begin{tabular}{|c|c|c|c|c|c|c|}
\hline VIH-Ascaphin-8 & VIHGFKDLLKGAAKALVKTVLF-NH ${ }_{2}$ & 0.978 & 0.932 & AMP & 0.999 & AMP \\
\hline CM15 & KWKLFKKIGAVLKVL & 0.845 & 0.948 & AMP & 0.998 & AMP \\
\hline GGH-CM15 & GGHKWKLFKKIGAVLKVL & 0.995 & 0.889 & AMP & 1.000 & AMP \\
\hline VIH-CM15 & VIHKWKLFKKIGAVLKVL & 0.992 & 0.993 & AMP & 1.000 & AMP \\
\hline CPF-ST3 & GLLGPLLKIAAKVGSNLL-NH ${ }_{2}$ & 0.982 & 1.000 & AMP & 0.998 & AMP \\
\hline GGH-CPF-ST3 & GGHGLLGPLLKIAAKVGSNLL-NH & 0.972 & 0.914 & AMP & 0.999 & AMP \\
\hline VIH-CPF-ST3 & VIHGLLGPLLKIAAKVGSNLL-NH & 0.982 & 0.995 & AMP & 0.999 & AMP \\
\hline Ponericin G6 & GLVDVLGKVGGLIKKLLP-NH & 0.945 & 0.874 & AMP & 0.997 & AMP \\
\hline GGH-Ponericin G6 & GGHGLVDVLGKVGGLIKKLLP-NH & 0.949 & 0.900 & AMP & 0.998 & AMP \\
\hline VIH-Ponericin G6 & VIHGLVDVLGKVGGLIKKLLP-NH & 0.973 & 0.942 & AMP & 0.996 & AMP \\
\hline Citropin1.1 & GLFDVIKKVASVIGGL-NH & 0.954 & 1.000 & AMP & 0.991 & AMP \\
\hline GGH-Citropin1.1 & GGHGLFDVIKKVASVIGGL-NH ${ }_{2}$ & 0.926 & 0.744 & AMP & 0.993 & AMP \\
\hline VIH-Citropin1.1 & VIHGLFDVIKKVASVIGGL-NH ${ }_{2}$ & 0.961 & 0.945 & AMP & 0.992 & AMP \\
\hline
\end{tabular}


Figure S1. Helical wheel projections and molecular models of AMPs used in this study. Helical wheel projections were obtained from HeliQuest server (http://heliquest.ipmc.cnrs.fr/) while molecular models of ATCUN-variants were built on the I-TASSER server using a hierarchical approach for peptide structure prediction.

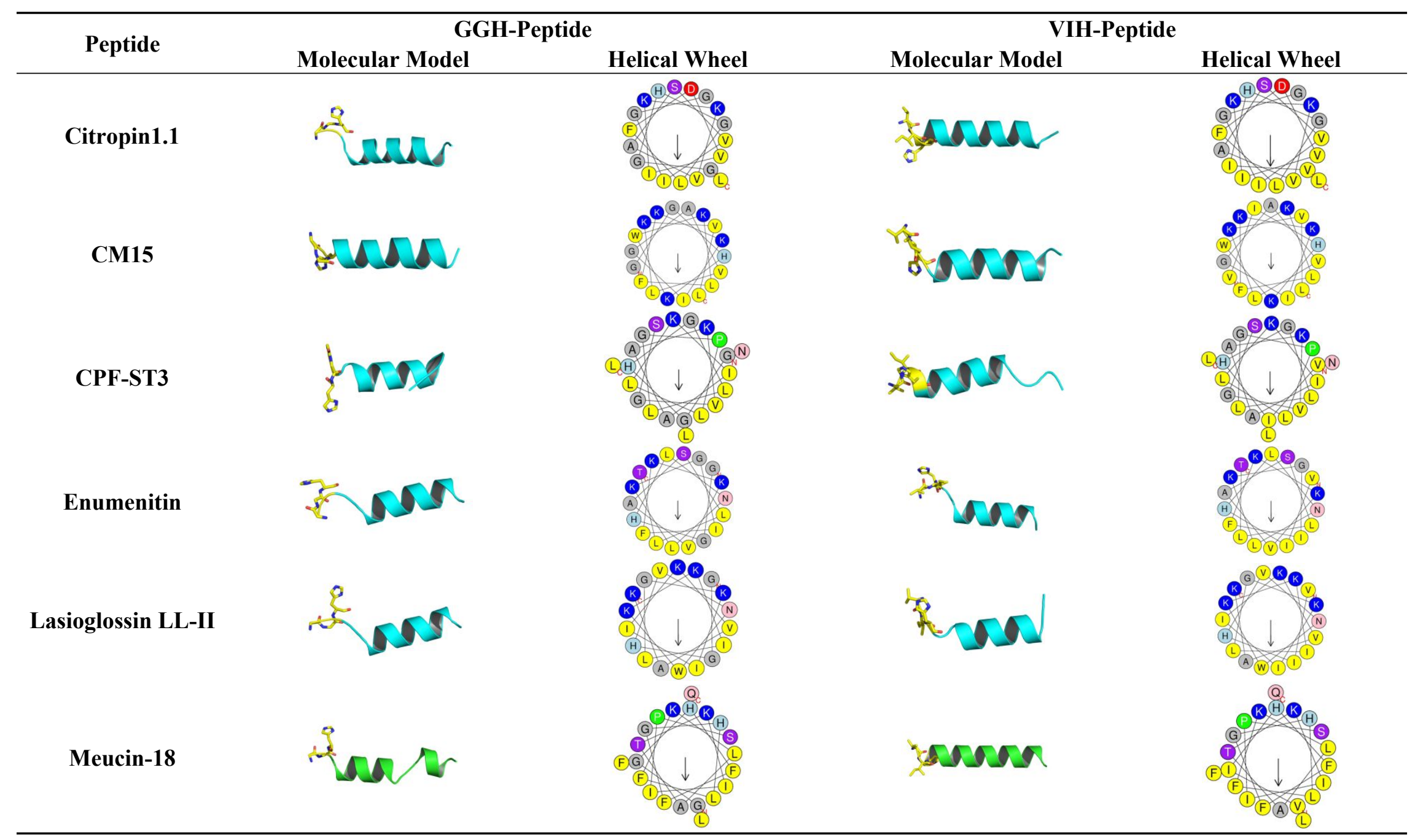




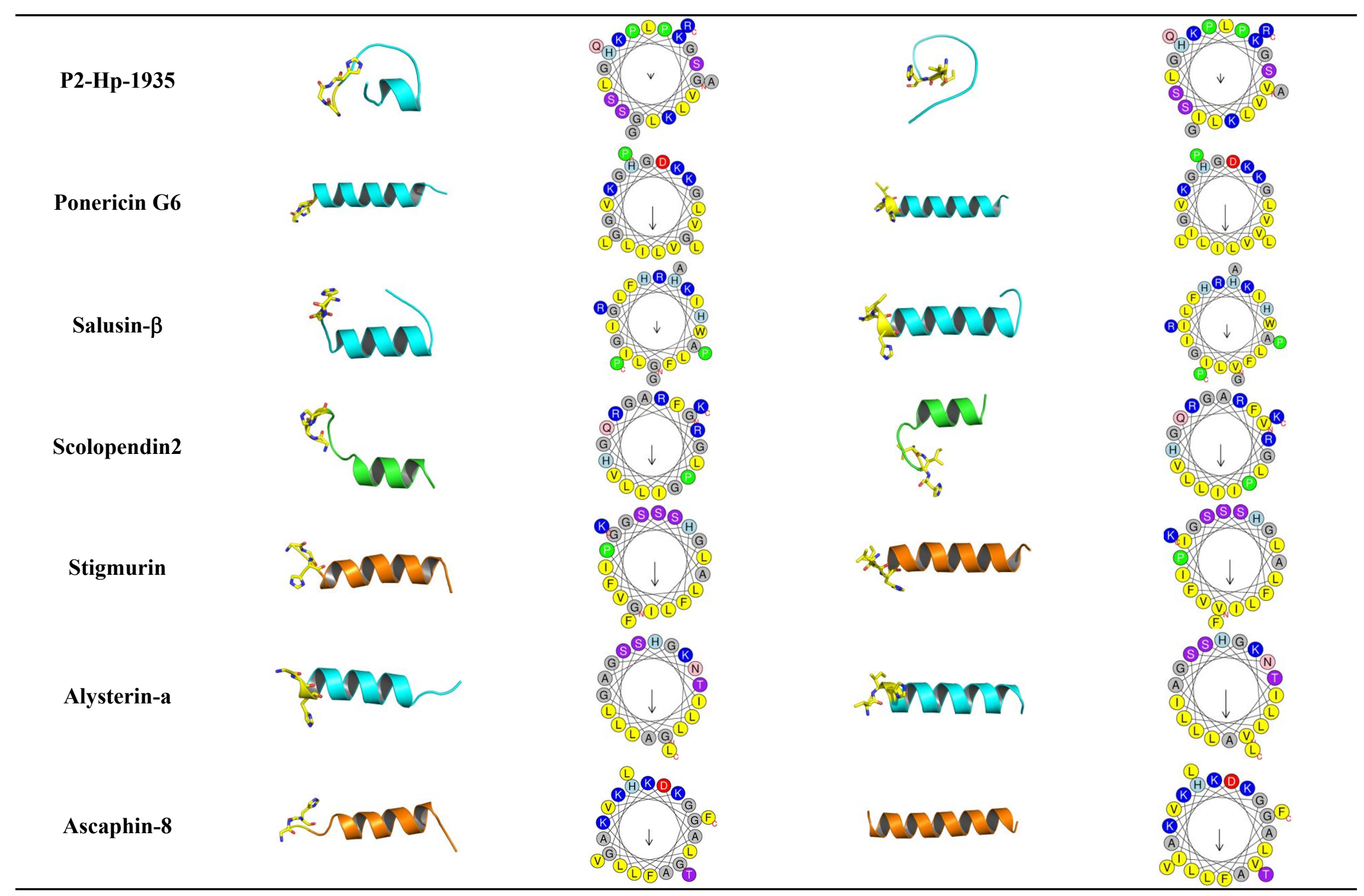




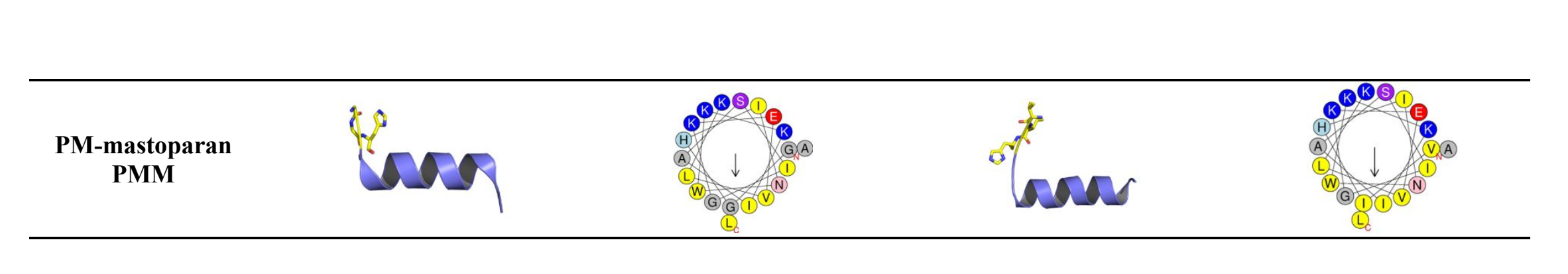


Table S3. Rank of AMPs based on the scores from helical wheel projection, molecular modelling and prediction algorithms. The prediction algorithm considered hydrophobicity, helicity and amphipathicity as criteria for its screening process.

\begin{tabular}{ccccc}
\hline Rank & Peptide & $\begin{array}{c}\text { Selical Tendency } \\
\text { in Wheel } \\
\text { Projection }\end{array}$ & $\begin{array}{c}\text { Prediction } \\
\text { Algorithms }\end{array}$ & $\begin{array}{c}\text { Molecular } \\
\text { Modelling }\end{array}$ \\
\hline 1 & Stigmurin & High & Low & High \\
2 & Scolopendin 2 & Low & Low & Low \\
3 & Salucin- $\beta$ & High & Low & Low \\
4 & P2-Hp-1935 & Low & Low & Low \\
5 & Pm-mastoparan PMM & High & High & High \\
6 & Meucin-18 & High & Low & Low \\
7 & Eumenitin & High & High & Low \\
8 & Lasioglossin LL-II & Low & High & High \\
9 & Alysterin-2a & High & Low & High \\
10 & Ascaphin-8 & High & High & Low \\
11 & CM15 & High & Low & High \\
12 & CPF-ST3 & Low & High & Low \\
13 & Ponericin G6 & High & Low & High \\
14 & Citropin 1.1 & High & Low & High \\
\hline
\end{tabular}

Table S4. Structure statistics for the tridimensional theoretical models generated by comparative modelling.

\begin{tabular}{cccc}
\hline Peptides & ProSA-web (z-score)* & G-factors** & Ramachandran (MFR - \%) $^{*}$ \\
\hline Citropin1.1 & 0.88 & 0.17 & 100 \\
GGH-Citropin1.1 & 0.17 & 0.24 & 100 \\
VIH-Citropin1.1 & 0.03 & -0.04 & 92.9 \\
GGH-CM15 & -1.14 & 0.0 & 92.9 \\
VIH-CM15 & -0.84 & 0.0 & 93.3
\end{tabular}

*All the z-score values are within the range observed for peptides studied by NMR and deposited in the PDB. **G-factors are within the expected values for reliable structures ( $>-0.5)$. MFR: most favorable regions. 


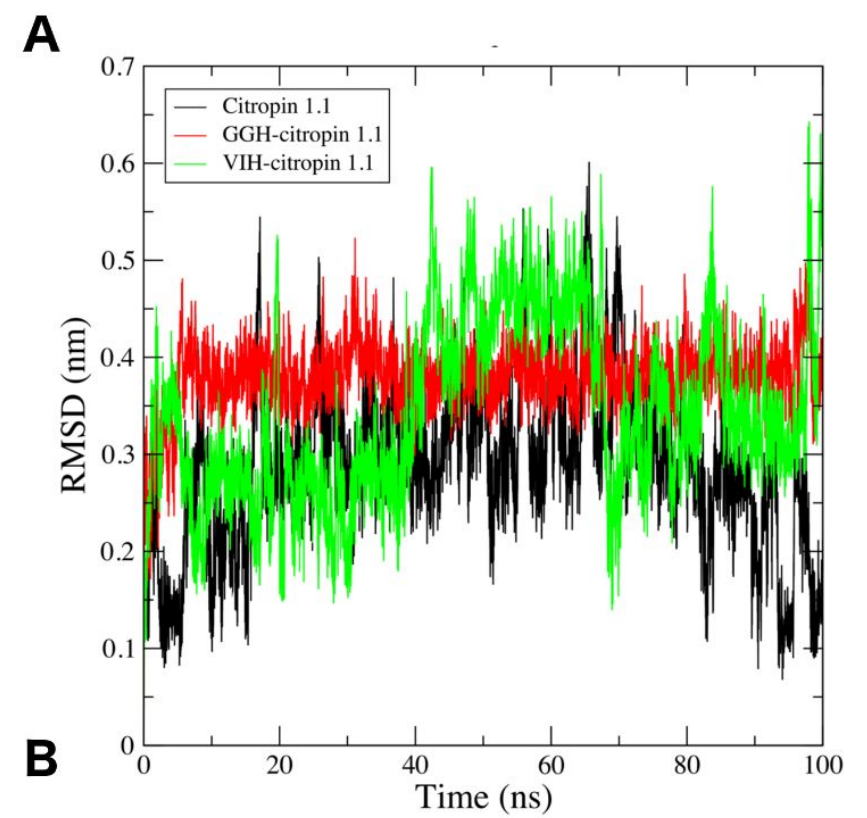

C
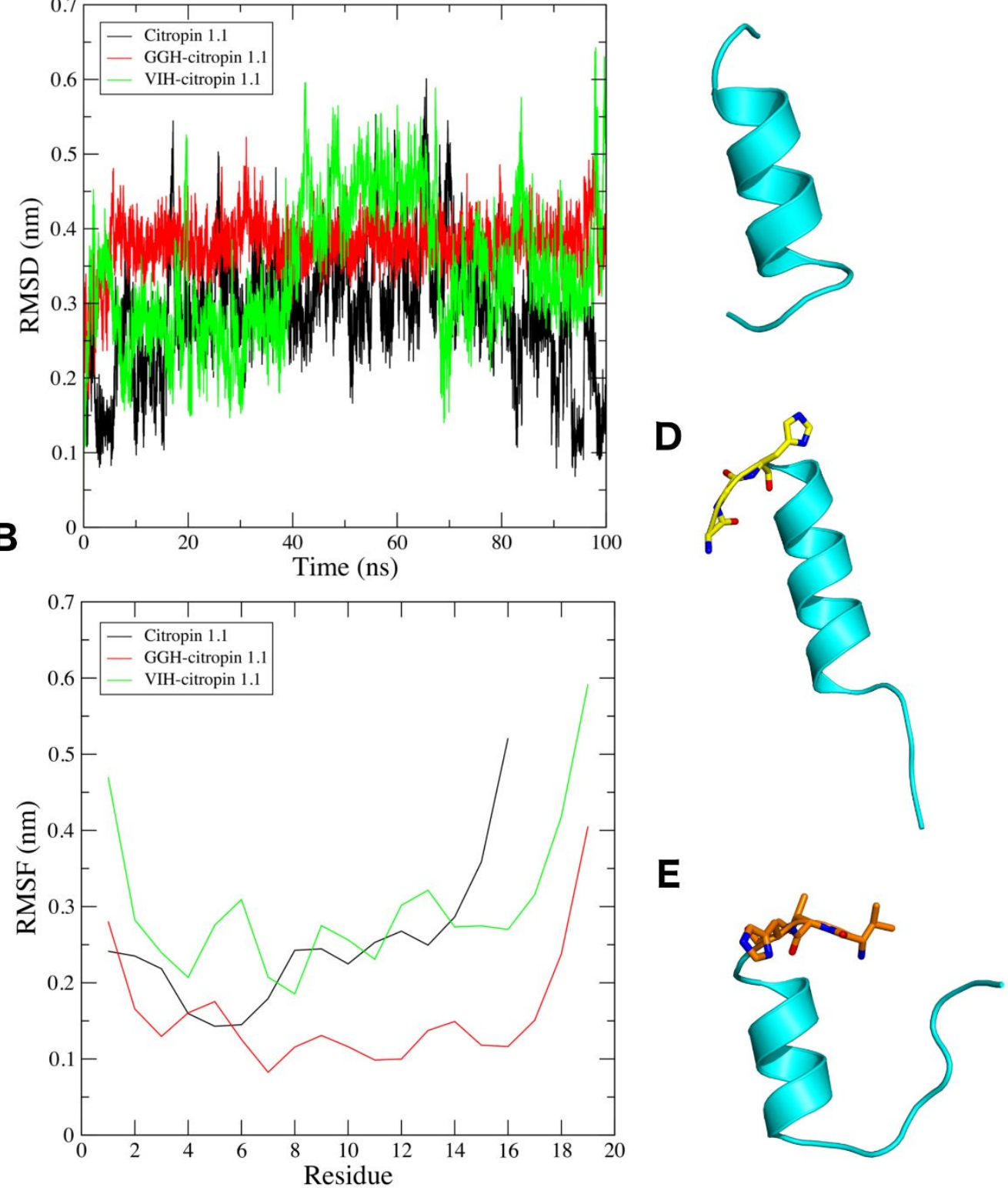

Figure S2. MD simulations of Citropin1.1 and its variants in TFE/water solution (1:1, v1v), during $100 \mathrm{ns.}$ The trajectory of the peptide was evaluated according to (A) the means of root mean square deviation (RMSD) and (B) the root mean square fluctuation (RMSF). The structural snapshots at $100 \mathrm{~ns}$ for (C) Citropin1.1, (D) GGH-Citropin1.1 and (E) VIH-Citropin1.1. Yellow sticks highlight the GGH motif in all cases; whereas orange sticks highlight the VIH motif. 

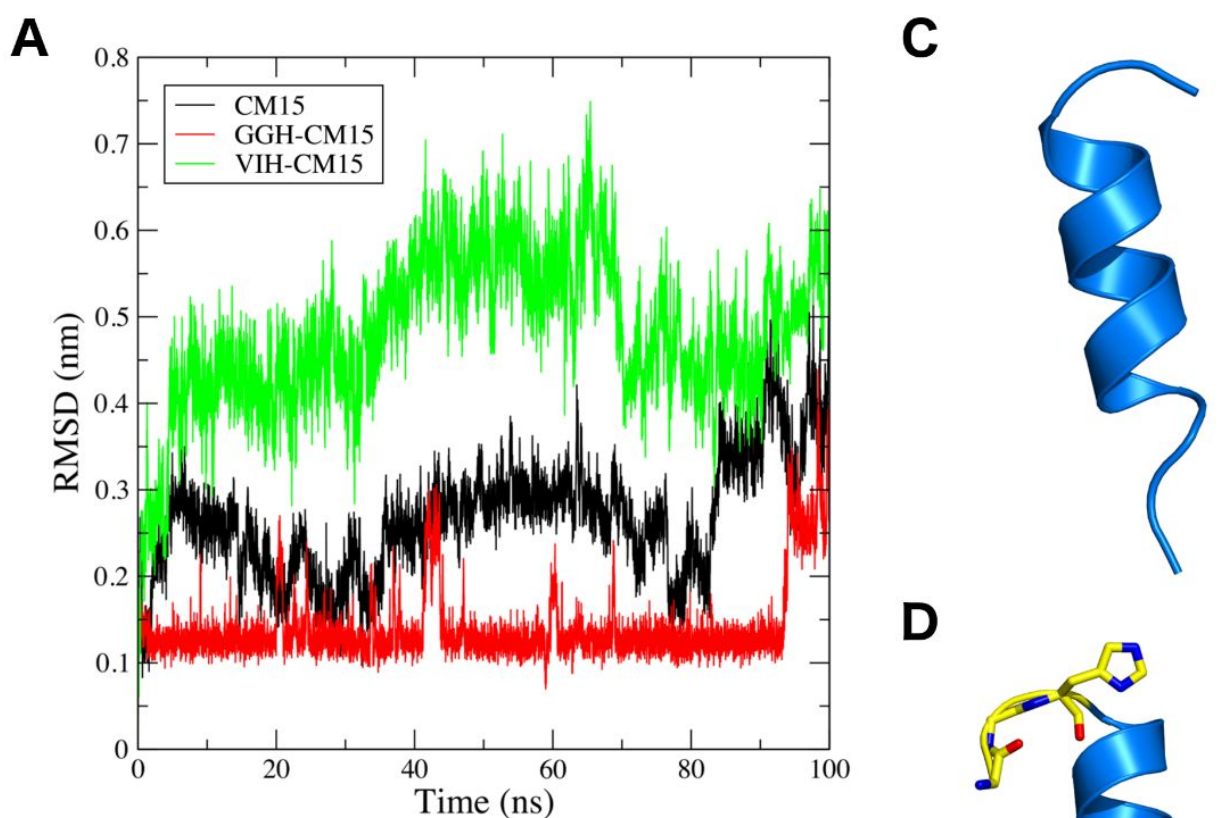

B
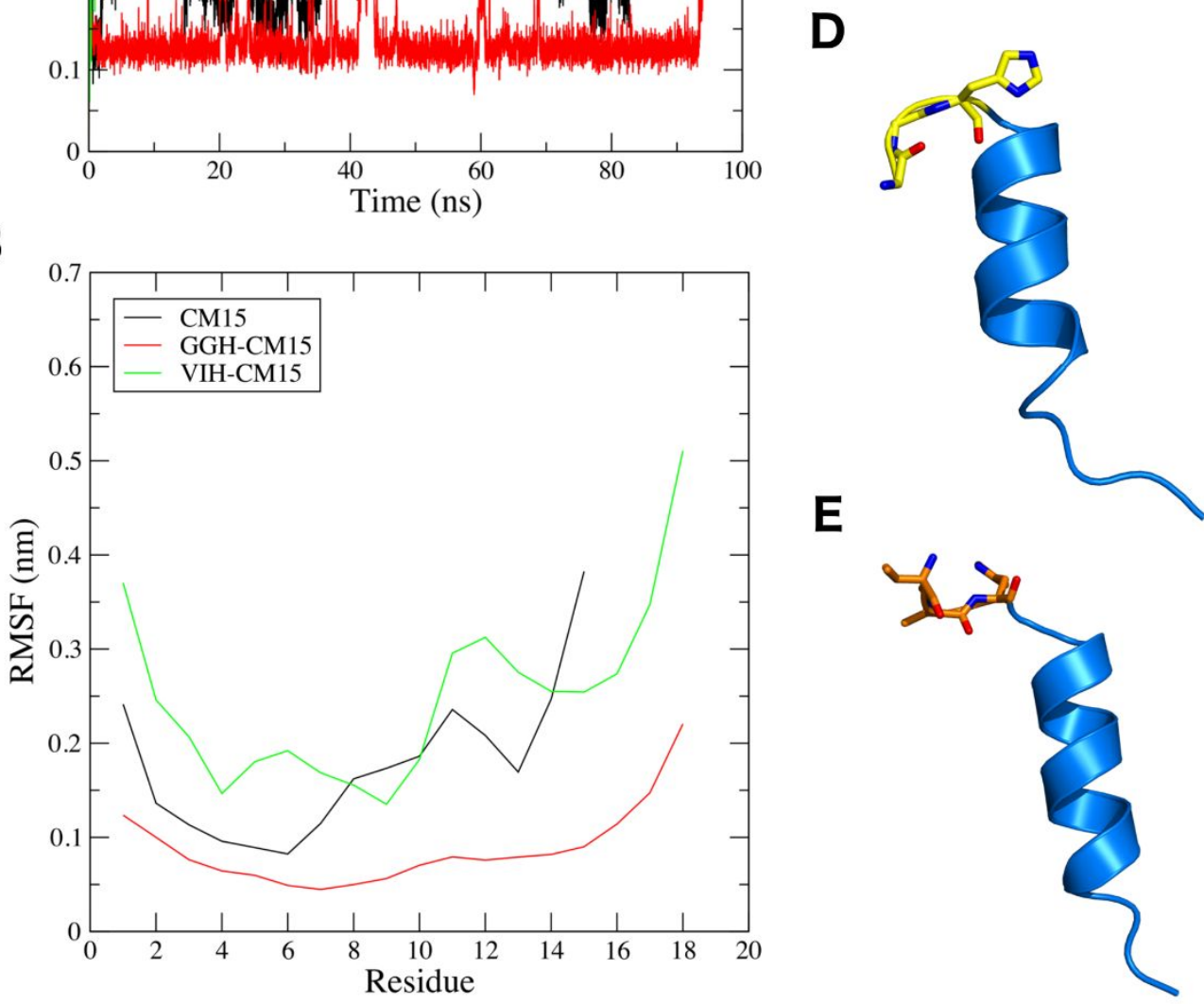

Figure S3. MD simulations of CM15 and its variants in TFE/water solution (1:1, v1v), during $100 \mathrm{~ns}$. The trajectory of the peptide was evaluated according to (A) the means of root mean square deviation (RMSD) and (B) the root mean square fluctuation (RMSF). The structural snapshots at $100 \mathrm{~ns}$ for (C) CM15, (D) GGH-CM15 and (E) VIH-CM15. Yellow sticks highlight the GGH motif in all cases; whereas orange sticks highlight the VIH motif. 

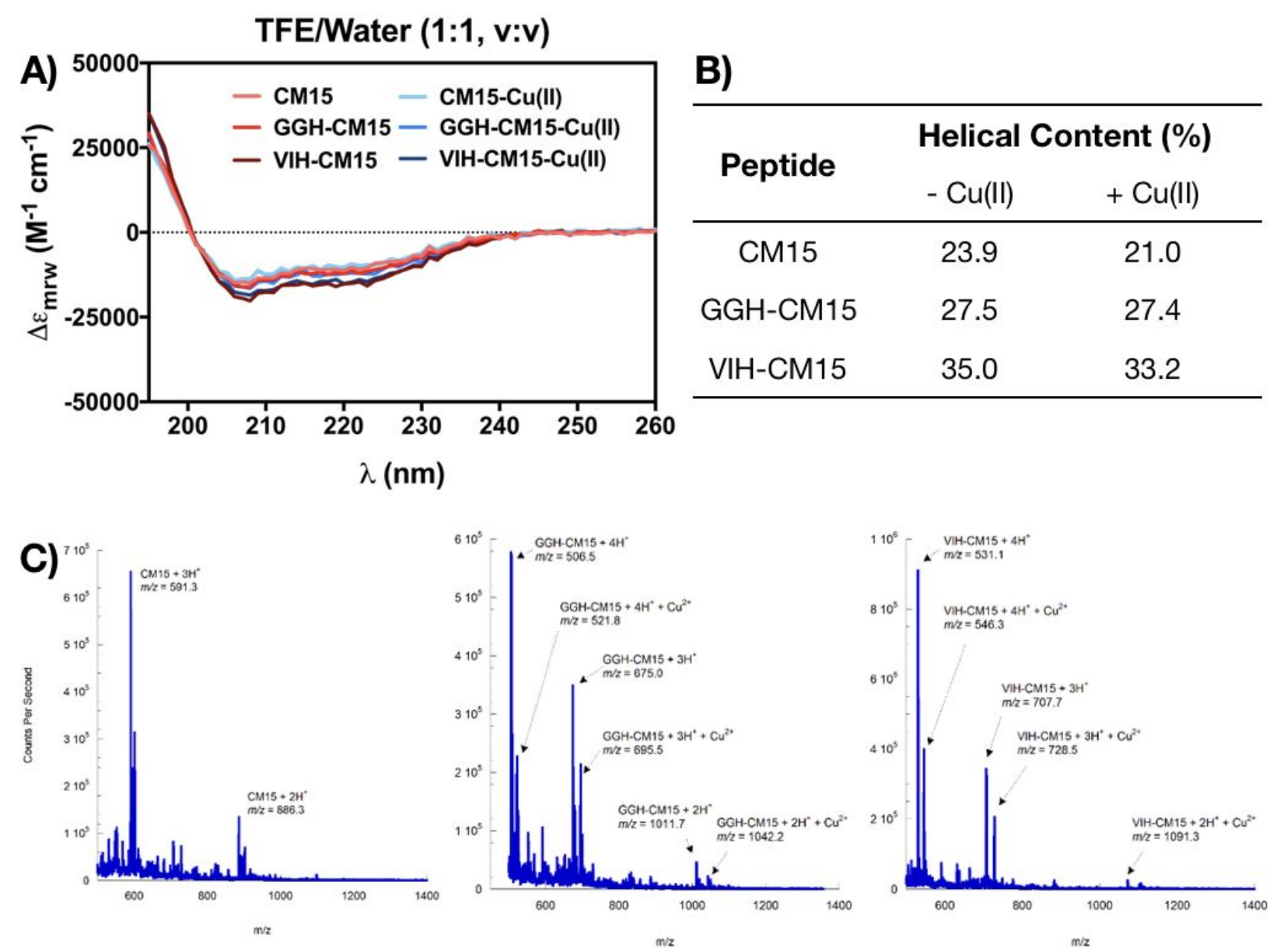

Figure S4. A) CD Spectra of CM15, GGH-CM15 and VIH-CM15, the peptide solutions at $50 \mu \mathrm{M}$ were obtained in the presence and absence of $\mathrm{Cu}(\mathrm{II})$ ion, on a Jasco J-710 spectropolarimeter in the far $\mathrm{UV}$ region with a quartz cuvette with a pathlength of $1 \mathrm{~mm}$ at room temperature, over five accumulations and a scan rate of $10 \mathrm{~nm} / \mathrm{min}$. The experiments were performed in a 50:50 mixture of nanopure water and 2,2,2trifluoroethanol (TFE). B) Alpha-helical content of CM15, GGH-CM15 and VIH-CM15 in the presence and absence of $\mathrm{Cu}$ (II) ions. C) Mass Spectra of CM15 (left), GGH-CM15 (middle), and VIH-CM15 (right) in the presence of $0.9 \mathrm{X} \mathrm{Cu}(\mathrm{II})$. 
Table S5. MICs of conventional antibiotics and the ATCUN-modified variants of CM15 and Citropin1.1 on the growth of planktonic E. coli (ATCC25922), E. coli (KpC1812446), K. pneumoniae (ATCC13883), and $K$. pneumoniae (KpC1825971) cells.

\begin{tabular}{lcccc}
\hline \multirow{2}{*}{$\begin{array}{l}\text { Antimicrobial } \\
\text { Agent }\end{array}$} & $\begin{array}{c}\text { E. coli } \\
\text { (KpC1812446) }\end{array}$ & $\begin{array}{c}\text { E. coli } \\
(\text { ATCC25922) }\end{array}$ & $\begin{array}{c}\text { K. pneumoniae } \\
(\text { ATCC13883) }\end{array}$ & $\begin{array}{c}\text { K. pneumoniae } \\
(\text { KpC1825971) }\end{array}$ \\
\hline Peptide & & & & \\
\hline CM15 & 5 & 5 & $>36$ & 8 \\
GGH-CM15 & 8 & 4 & $>32$ & 8 \\
VIH-CM15 & 4 & 4 & 30 & ND \\
Citropin1.1 & $>40$ & ND & $>40$ & ND \\
GGH-Citropin1.1 & $>34$ & ND & $>34$ & ND \\
VIH-Citropin1.1 & $>33$ & ND & $>33$ & \\
\hline Antibiotic & & & & \\
\hline MER & 0.6 & & $>167$ & \\
AmP & $>183$ & & $>183$ & \\
TRI & $>220$ & & $>198$ & \\
CHL & $>198$ & & $>110$ & \\
STR & 14 & & & \\
\hline ND - nt & & & & \\
\hline
\end{tabular}

ND - not determined, MER - meropenem, AmP - ampicillin, TRI - trimethoprim, STR - streptomycin and CHL - chloramphenicol.

Table S6. MBICs of conventional antibiotics and ATCUN-variants of CM15 and Citropin1.1 on the biofilm formation of E. coli (ATCC 25922), E. coli (KpC1812446), K. pneumoniae (ATCC13883), and K. pneumoniae (KpC1825971) cells.

\begin{tabular}{ccccc}
\hline \multirow{2}{*}{ Peptide } & \multicolumn{3}{c}{ MBIC $(\boldsymbol{\mu M M})$} \\
\cline { 2 - 5 } & $\begin{array}{c}\text { E. coli } \\
(\text { KpC1812446) }\end{array}$ & $\begin{array}{c}\text { E. coli } \\
\text { (ATCC 25922) }\end{array}$ & $\begin{array}{c}\text { K. pneumoniae } \\
\text { (ATCC13883) }\end{array}$ & $\begin{array}{c}\text { K. pneumoniae } \\
\text { (KpC1825971) }\end{array}$ \\
\hline CM15 & 9 & 5 & $>36$ & 5 \\
GGH-CM15 & 4 & 4 & $>32$ & 4 \\
VIH-CM15 & 8 & 4 & $>30$ & 4 \\
Citropin1.1 & $>40$ & ND & $>40$ & ND \\
GGH-Citropin1.1 & $>34$ & ND & $>34$ & ND \\
VIH-Citropin1.1 & $>33$ & ND & $>33$ & ND \\
\hline Antibiotic & & & & ND \\
\hline MER & 0.6 & ND & $>167$ & ND \\
AmP & $>183$ & ND & $>183$ & ND \\
TRI & $>220$ & ND & $>220$ & ND \\
CHL & $>198$ & ND & $>198$ & $>110$ \\
STR & 14 & ND & N
\end{tabular}

ND-not determined. MER- meropenem, AmP- ampicillin, TRI-trimethoprim, STR-streptomycin and CHL- chloramphenicol 
Table S7. FICI for combinations of conventional antibiotics and CM15 variants for inhibition of biofilm formation by E. coli (KpC 1812446).

\begin{tabular}{|c|c|c|c|c|c|}
\hline \multirow[b]{2}{*}{ Antibiotic } & \multicolumn{3}{|c|}{ MBIC $(\mu \mathrm{M})$} & \multirow[b]{2}{*}{ FICI } & \multirow[b]{2}{*}{ Interaction } \\
\hline & $\begin{array}{c}\text { Antibiotic } \\
\text { alone }\end{array}$ & $\begin{array}{c}\text { Antibiotic }+ \\
\text { Peptide }\end{array}$ & $\begin{array}{l}\text { Peptide }+ \\
\text { antibiotic }\end{array}$ & & \\
\hline \multicolumn{6}{|c|}{ CM15 } \\
\hline MER & 0.6 & 0.16 & 0.56 & 0.33 & Synergy \\
\hline $\mathrm{AmP}$ & 366 & 366 & 2 & 1.2 & Indifferent \\
\hline TRI & 440 & 440 & 9 & 2 & Indifferent \\
\hline CHL & 396 & 396 & 9 & 2 & Indifferent \\
\hline STR & 14 & 4 & 2 & 0.46 & Synergy \\
\hline \multicolumn{6}{|c|}{ GGH-CM15 } \\
\hline MER & 0.6 & 0.013 & 1 & 0.27 & Synergy \\
\hline $\mathrm{AmP}$ & 366 & 366 & 4 & 2 & Indifferent \\
\hline TRI & 440 & 220 & 4 & 1.5 & Indifferent \\
\hline CHL & 396 & 396 & 4 & 2 & Indifferent \\
\hline STR & 14 & 4 & 2 & 0.75 & Additive \\
\hline \multicolumn{6}{|c|}{ VIH-CM15 } \\
\hline MER & 0.6 & 0.04 & 1 & 0.19 & Synergy \\
\hline $\mathrm{AmP}$ & 366 & 366 & 4 & 1.5 & Indifferent \\
\hline TRI & 440 & 220 & 4 & 1 & Indifferent \\
\hline CHL & 396 & 396 & 3 & 1.38 & Indifferent \\
\hline STR & 14 & 7 & 4 & 1 & Indifferent \\
\hline
\end{tabular}

Table S8. FICI for the combinations of conventional antibiotics and CM15 variants at inhibiting E. coli (KpC 1812446) planktonic cells.

\begin{tabular}{|c|c|c|c|c|c|}
\hline \multirow[b]{2}{*}{ Antibiotic } & \multicolumn{3}{|c|}{ MBIC $(\mu \mathrm{M})$} & \multirow[b]{2}{*}{ FICI } & \multirow[b]{2}{*}{ Interaction } \\
\hline & $\begin{array}{c}\text { Antibiotic } \\
\text { alone }\end{array}$ & $\begin{array}{c}\text { Antibiotic + } \\
\text { Peptide }\end{array}$ & $\begin{array}{l}\text { Peptide }+ \\
\text { antibiotic }\end{array}$ & & \\
\hline \multicolumn{6}{|c|}{ CM15 } \\
\hline MER & 0.6 & 0.6 & 2 & 1.4 & Indifferent \\
\hline AmP & 366 & 366 & 4 & 1.8 & Indifferent \\
\hline TRI & 440 & 55 & 2 & 0.525 & Synergy \\
\hline CHL & 396 & 0.8 & 4 & 0.802 & Additive \\
\hline STR & 14 & 4 & 2 & 0.69 & Additive \\
\hline \multicolumn{6}{|c|}{ GGH-CM15 } \\
\hline MER & 0.6 & 0.16 & 2 & 0.52 & Synergy \\
\hline AmP & 366 & 366 & 4 & 1.5 & Indifferent \\
\hline TRI & 440 & 110 & 2 & 0.5 & Synergy \\
\hline $\mathrm{CHL}$ & 396 & 3 & 4 & 0.5008 & Synergy \\
\hline STR & 14 & 14 & 4 & 1.5 & Indifferent \\
\hline \multicolumn{6}{|c|}{ VIH-CM15 } \\
\hline MER & 0.6 & 0.3 & 2 & 1 & Additive \\
\hline $\mathrm{AmP}$ & 366 & 366 & 4 & 2 & Indifferent \\
\hline TRI & 440 & 220 & 2 & 1 & Additive \\
\hline CHL & 396 & 0.8 & 4 & 1.002 & Indifferent \\
\hline STR & 14 & 0.4 & 4 & 1.29 & Indifferent \\
\hline
\end{tabular}

